\title{
A Comparison between the Kinematic Effects of Knee Orthosis and Ankle-Foot Orthosis in Knee Varus Alignment
}

\author{
Sol Rim Kim ${ }^{1}$ Jang Hwan Kim² and Kang Seong Lee ${ }^{3 *}$ \\ ${ }^{1}$ Dept. of Rehabilitation Science Graduate School, Hanseo University, Korea \\ ${ }^{2,3}$ Dept. of Biomedical Engineering Welfare Technology, Hanseo University, Korea \\ 1profpo@hanseo.ac.kr, ${ }^{2}$ ksr9029@angel-robotics.com, ${ }^{3}$ cpo114@hanseo.ac.kr
}

\begin{abstract}
This study aimed to compare the effects of a knee brace and ankle-foot orthosis on knee osteoarthritis. The study included 13 adult subjects with a knee varus exceeding 50 mm between the medial epicondyles of the femurs. Each subject participated in 3 walking conditions. In this study, only the knee orthosis reduced knee varus angle in patients with knee osteoarthritis. Therefore, we expect that newly applied ankle-foot orthosis will increase the diversity of conservative treatments for knee osteoarthritis, along with typically used conventional knee orthosis.
\end{abstract}

Keywords: Knee orthosis, Ankle-foot orthosis, Varus knee, Kinematic, Osteoarthritis

\section{Introduction}

Osteoarthritis is the most common joint disease and is characterized as non-repaired damage to the joint structures including cartilage, bone, and joint capsules [1]. Osteoarthritis of the knee joint is one of the most common causes of elderly population disorders [2]. It has been reported that $6 \%$ of adults older than 30 years of age (about 9,700,000 people) suffer from symptoms of knee osteoarthritis [3].

The use of orthosis as a conservative treatment for knee osteoarthritis not only delays the progression of the disease, but also decreases the timing of knee pronation while reducing pain and maintaining knee function. As such, the goal of orthosis is to reduce the moment of knee pronation [4].

Moyer et al. [5] compared the effects of a knee brace and lateral wedge insole combination in 16 patients with knee osteoarthritis. In this study, the knee brace was associated with a greater reduction in knee adduction than the lateral wedge insole and the reduction in adduction moment was most efficient once the knee brace and lateral wedge insole were applied together. Pagani et al. [6] created ankle-foot orthosis for a knee osteoarthritis patient and compared this with lateral wedge insoles to assess adduction timing in the knees of 14 adults with knee varus. The patient with ankle-foot orthosis, which was adjusted to valgus varus, had neutral delayed adduction of the knee compared to those without the brace. There were no significant differences in the three adjustments, and the lateral wedge insole did not reduce the knee adduction timing.

Most studies in the field compare lateral wedge and knee brace related to knee osteoarthritis, but no studies have compared the effects of knee braces and ankle-foot orthosis when used

Article history:

Received (July 21, 2019), Review Result (August 26, 2019), Accepted (October 19, 2019) 
together. As such, this study aimed to compare the effects of knee brace and ankle-foot orthosis for the conservative treatment of patients with knee osteoarthritis.

\section{Methods}

\subsection{Subjects}

In this study, 13 adults (10 males, 3 females) with no medical issues participated instead of patients having knee osteoarthritis, and they had varus knees at certain level. The criteria for subject inclusion in this study were as follows.

First, the distance between the femur and the intercondylar as measured with calipers in the standing position needed to be equal or greater than $50 \mathrm{~mm}$ [7]. Second, the subject had no knee injuries, knee surgeries, or walking abnormalities in the past year. Third, the subject understood the study and signed the declaration. Fourth, the subjects needed to be college students in their early 20 's as $21.6 \pm 2.43$ in average age and not actively exercising.

\subsection{The brace}

The ankle- foot orthosis (AFO) used in the study was Agilium Freestep (Ottobock, Duderstadt, Germany).

The knee orthosis (KO) used was the OA Every day (Donjoy Orthopedics, CA, USA).
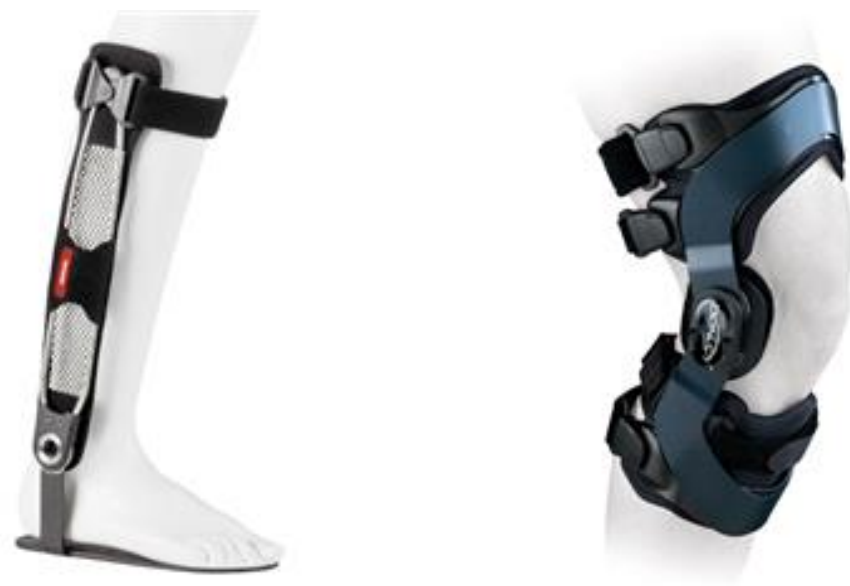

Figure 1. Ankle-Foot Orthosis (AFO)and Knee Orthosis (KO)

\subsection{Preparation of the subjects}

Vicon (Vicon motion system Ltd, Oxford, UK), a three-dimensional motion assessment device with an infrared camera, was used for gait analysis. Six cameras were used, and the sampling rate was $150 \mathrm{~Hz}$. The marker in system was Plug in gait. A $14 \mathrm{~mm}$ diameter infrared reflecting marker was attached to the anterior-superior and posterior-superior iliac spines, lateral epicondyle of the knee, lateral malleoli, the thigh, the lower leg, the second metatarsal head, and posterior calcaneus. The markers were attached to the corresponding sites on the brace if the brace covered the sites and adjusted to body size accordingly. 


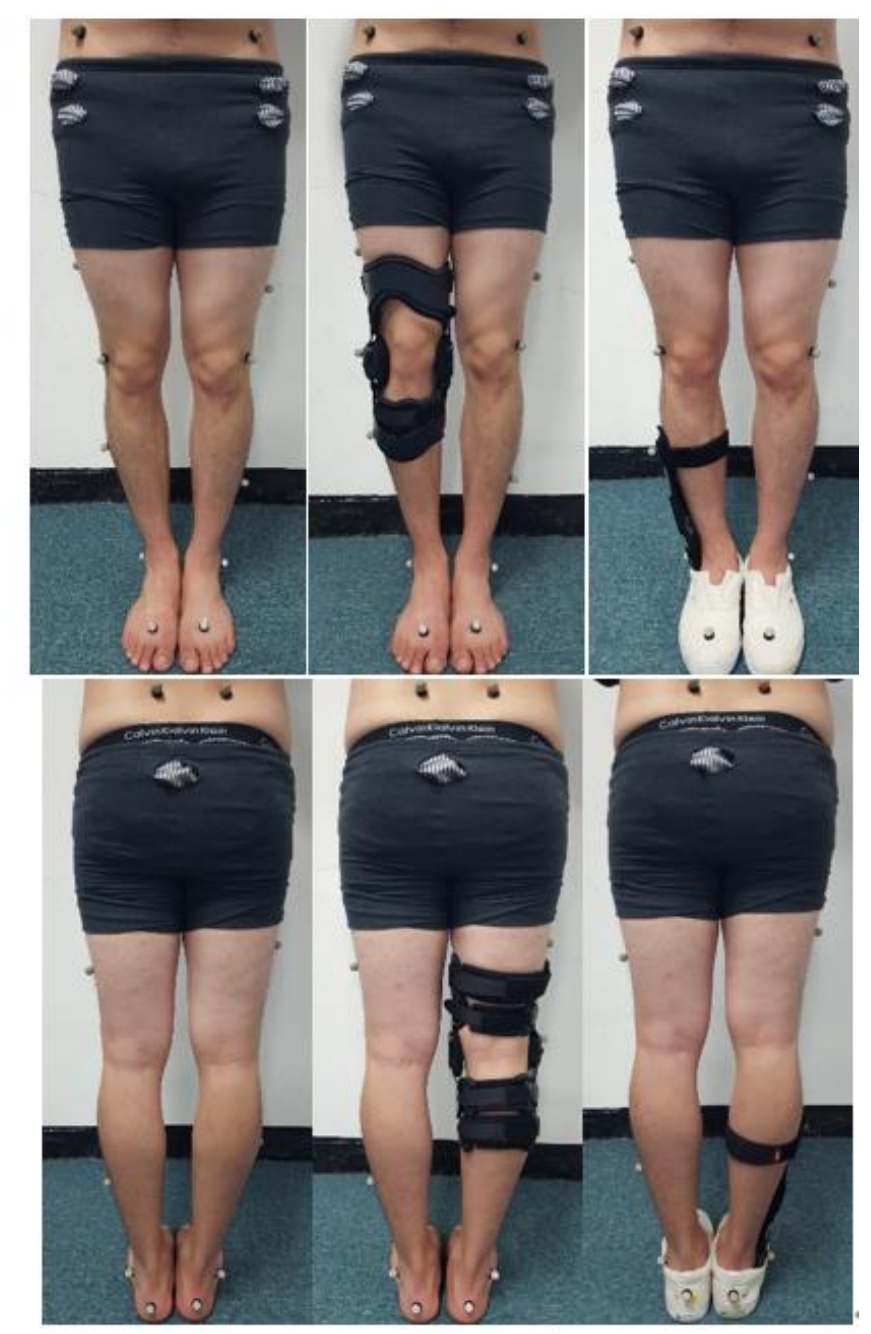

Figure 2. Anatomical landmarks for the attachment of infrared reflecting markers

\subsection{Experimental design}

The subjects were given prepared clothes and slippers on arrival and they underwent gait analysis whilst wearing the brace for 2 hours with a 20 min break after each experiment. There were three experimental conditions: no brace, knee brace only, and ankle-foot orthosis. The order of investigation was randomized. Ankle-foot orthosis were applied whilst wearing the shoes in the instruction manual, and an insole was fitted to the opposing foot to elevate it to a similar level. This was repeated three times for each condition.

\subsection{Statistical analysis}

One-way repeated ANOVA was performed to compare the data collected in gait analysis, and Bonferroni analysis was performed for post-hoc analysis. SPSS (IBM Corp, NW, US) 18.0.0 version was used for all statistical analysis, and statistical significance levels were set to $\mathrm{p}<0.05$. 


\section{Results}

\subsection{General characteristic of subjects}

Subjects are 13 and populated as 10 males and 3 females. The average of the age is $21.62 \pm 2.43$, the average of the height is $172.38 \pm 5.92 \mathrm{CM}$, the average of the weight is $63.92 \pm 9.06 \mathrm{~kg}$, and the distance of the femoral condyles is $55.70 \pm 4.91 \mathrm{~mm}$ [Table 1].

Table 1. General characteristic of subjects $(\mathrm{n}=13)$

\begin{tabular}{|c|c|}
\hline & Mean \pm SD \\
\hline Age & $21.62 \pm 2.43$ \\
\hline Height $(\mathrm{cm})$ & $172.38 \pm 5.92$ \\
\hline Weight(kg) & $63.92 \pm 9.06$ \\
\hline $\begin{array}{c}\text { The distance between the femur and the } \\
\text { intercondylar(mm) }\end{array}$ & $55.70 \pm 4.91$ \\
\hline
\end{tabular}

\subsection{Characteristic of the knee in the frontal plane}

\subsubsection{Relationship between the peak of adduction moment and the inversion angle}

The knee adduction moment measured at the first peak showed significant differences between the three conditions at $4.45 \pm 3.55^{\circ}$ without the brace, $2.37 \pm 3.92^{\circ}$ with the knee brace,

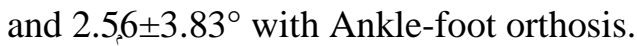

In post analysis, there was significant difference between the condition without the brace and with Ankle-foot orthosis, but no difference between no brace and ankle-foot orthosis or between the knee brace and ankle-foot brace [Table 2].

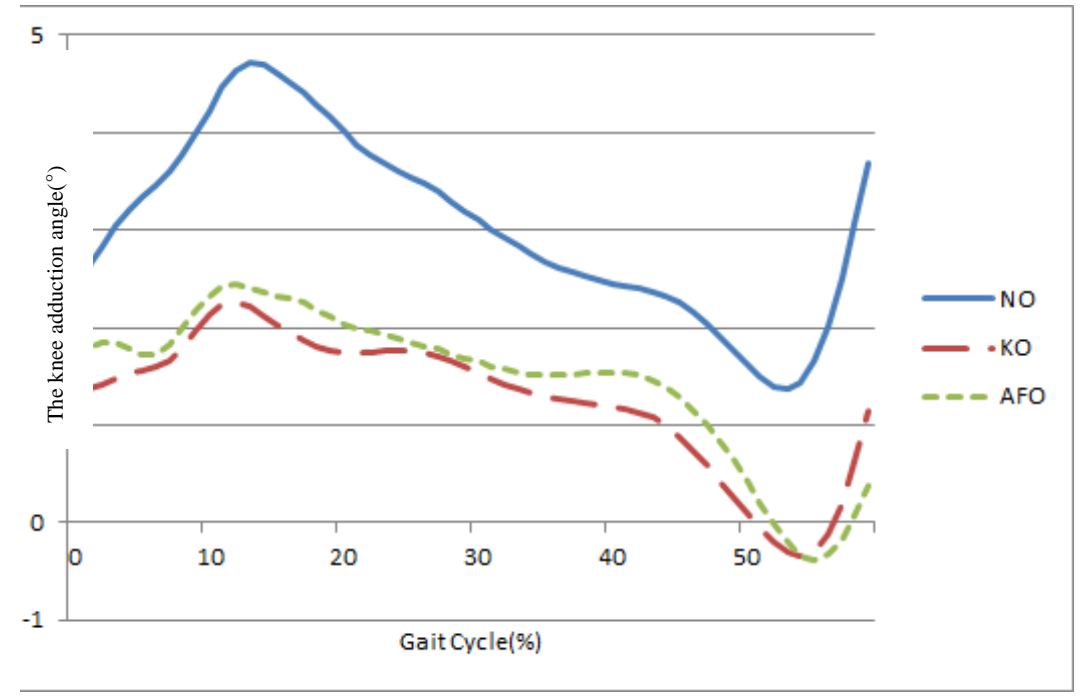

Figure. 3 The knee adduction angle 
Table 2. KAM-1st the knee adduction angle at the peak

\begin{tabular}{|c|c|c|c|c|c|}
\hline & Condition & Average & Standard deviation & $\mathrm{F}$ & $\mathrm{p}$ \\
\hline \multirow{2}{*}{${ }^{\mathrm{a}} \mathrm{KVA}(\mathrm{deg})$} & ${ }^{\mathrm{b}} \mathrm{NO}$ & $4.45 \dagger$ & 3.55 & - & - \\
\cline { 2 - 6 } & ${ }^{\mathrm{c}} \mathrm{KO}$ & $2.37 \S$ & 3.92 & 3.635 & 0.029 * \\
\cline { 2 - 6 } & $\mathrm{d} \mathrm{AFO}$ & 2.56 & 3.83 & - & - \\
\hline
\end{tabular}

aKVA(knee varus angle), bNO(no orthosis), cKO(knee orthosis), dAFO(ankle-foot orthosis), §, significant difference compared with the non-brace condition; + , significant difference compared with the knee brace condition.

\subsection{Characteristics of the ankle in the frontal plane}

The knee adduction moment and ankle inversion angle at the first peak were not significantly different among the three conditions of $2.69 \pm 1.61^{\circ}$ without the brace, $2.35 \pm 1.59^{\circ}$ with the knee brace, and $2.36 \pm 1.56^{\circ}$ with ankle-foot orthosis [Table 3].

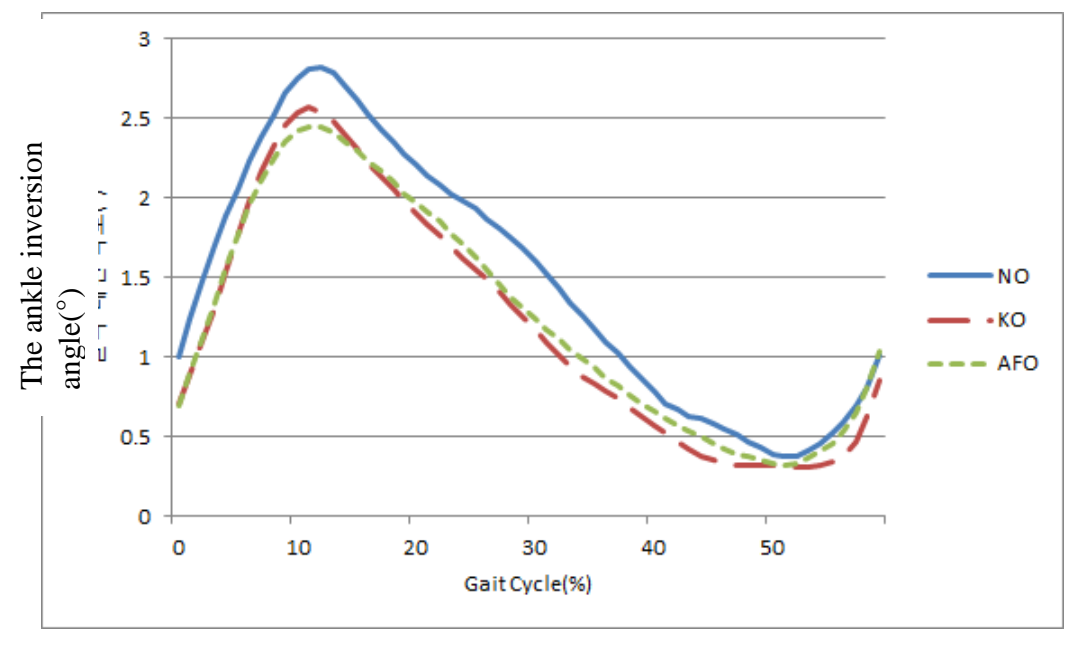

Figure 4. The ankle inversion angle

Table 3. KAM-1st ankle inversion angle at the first peak

\begin{tabular}{|c|c|c|c|c|c|}
\hline & Condition & Average & Standard deviation & $\mathrm{F}$ & $\mathrm{p}$ \\
\hline \multirow{3}{*}{${ }^{\mathrm{a}} \mathrm{AII}(\mathrm{deg})$} & ${ }^{\mathrm{b}} \mathrm{NO}$ & 2.69 & 1.61 & - & - \\
\cline { 2 - 6 } & ${ }^{\mathrm{c}} \mathrm{KO}$ & 2.35 & 1.59 & 0.582 & 0.560 \\
\cline { 2 - 6 } & $\mathrm{d} \mathrm{AFO}$ & 2.36 & 1.56 & - & - \\
\hline
\end{tabular}

aAIA(ankle inversion angle), bNO(no orthosis), cKO(knee orthosis), dAFO(ankle-foot orthosis)

\section{Discussion}

The purpose of this study was to compare the kinematic effects of knee osteoarthritis with a currently used knee brace and newly applied ankle-foot orthosis in 13 patients. 
The knee varus angle measured at the moment of the first peak of the moment of knee adduction was $4.45 \pm 3.55^{\circ}$ without the brace, $2.37 \pm 3.92^{\circ}$ with the knee brace, and $2.56 \pm 3.83^{\circ}$ with ankle-foot orthosis. There was a significant decrease in the knee varus angle for the applied knee brace compared to the when no brace was applied, but no significant difference was shown between no brace and ankle-foot orthosis, or between knee brace and ankle-foot orthosis conditions. In a previous study by Pagani et al, the use of a knee brace showed a reduction in the knee adduction moment in 10 osteoarthritis patients when compared to use of lateral wedge insoles[8]. This study found similar results as a significant reduction in knee varus angle with the valgus knee brace was seen. Pagani et al. [6] compared a lateral wedge insole with anklefoot orthosis for the reduction of knee adduction moment. In this study, the use of ankle-foot orthosis was not associated with a reduction in knee varus angle compared when no brace was used. And, decreased knee adduction moment in significant reducing knee varus angle under applied knee brace is presented as slightly larger than applied ankle-foot orthosis so that, the decreased knee varus angle may be affected to the value of knee adduction moment.

The inversion angle of the ankle measured at the first peak in the knee adduction moment was not significantly different among the three conditions. It was $2.69 \pm 1.61^{\circ}$ without the brace, $2.35 \pm 1.59^{\circ}$ with knee brace, and $2.36 \pm 1.56^{\circ}$ with ankle-foot orthosis. A study by Pagani et al comparing a lateral wedge insole and ankle-foot orthosis [6] showed that the angle of the ankle in the frontal plane was significantly decreased with lateral wedge insole as compared to when no brace was used. However, it was not significantly different between lateral wedge insole and ankle-foot orthosis. The knee adduction moment was not affected with lateral wedge insole as compared to when no brace was used. In contrast, the use of ankle-foot orthosis did produce a significant effect. This was because there was no change in the position of the tibia and the knee angle even though the ankle was in a significantly different position in the frontal plane. In this study, the knee brace and ankle-foot orthosis had no significant difference on the ankle inversion angle as compared to when no brace was used, but they were effective in reducing the knee adduction moment. Therefore, the angle of the ankle in the frontal plane had no significant effect on the knee angle and adduction moment.

\section{Conclusion}

This study compared the kinematic characteristics of 13 adults with a varus of greater than $50 \mathrm{~mm}$ between the intercondylar of the femur in the knee during the following three conditions: no brace, knee brace, and ankle-foot orthosis.

First, compared to the no brace condition, ankle-foot orthosis for knee osteoarthritis had no significant effect on the varus angle in the stance phase.

Second, there were significant differences in the varus angle of the knee with the knee brace during the stance phase as compared to when no brace was used.

Third, there were no differences in the varus angle of the knee between the no brace and ankle-foot orthosis groups.

In conclusion, the new ankle-foot orthosis for knee osteoarthritis does not reduce the varus angle of the knee compared to the existing knee brace. However, this study demonstrates that use of knee brace reduces the varus angle in knee varus alignment as compared to when no brace is used.

\section{References}

[1] D. T. Felson, Clin. Orthop. Relat. Res, vol.427, pp.16-21, (2004) 
[2] A. A. Guccione, D. T. Felson, J. J. Anderson, J. M . Anthony, Y. Zhang, P. W. Wilson, M. Kelly-Hayes, P. A. Wolf, B. E. Keger, and W. B. Kannel, Am., J. Public Health, vol.84, pp.351-358, (1994)

[3] D. T. Felson, R. C. Lawrence, P. A. Dieppe, R. Hirsch, C. G Helmick, J. M. Jordan, R. S. Kington, N. E. Lane, M. C. Nevitt, Y. Zhang, M. Sowers, T. McAlindon, T. D. Spector, A. R. Poole, S. Z. Yanovski, G. Ateshian, L. Sharma, J. A. Buckwalter, K. D. Brandt, and J. F. Fires, Ann. Intern. Med, vol.133, pp.635-646, (2000)

[4] R. K. Jones, C. J. Nester, J. D. Richards, W. Y. Kim, D. S. Johnson, S. J. P. Laxton, and S. F. Tyson, Gait Posture, vol.37, pp.368-372, (2003)

[5] R. F. Moyer, T. B. Birmingham, C. E. Dombroski, R. F. Walsh, K. M Leitch, T. R. Jenkyn, and J. R. Jiffin, Arch. Phys. Med. Rehabil, vol.94, pp.103-112, (2013)

[6] C. H. F. Pagani, S. Willwacher, and R. Benker, Brüggermann, Prosthet. Orthot. Int. vol.38, pp.481-491, (2014)

[7] A. M. Navali, L. A. S. Bahari, and B. Nazari, Sports. Med. Arthrosc. Rehabil. Ther. Technol. vol.4, no.40, (2012)

[8] C. H. F. Pagani, M. Hinrichs, and G. P. Brüggermann, J. Orthop. Res. vol.30, pp.1125-1132, (2012) 
A Comparison between the Kinematic Effects of Knee Orthosis and Ankle-Foot Orthosis in Knee Varus Alignment

This page is empty by intention. 